\title{
Universal Grey Method Based on Hyper-chaos for Solving Forward Displacement of Generalized Parallel Mechanism
}

\author{
http://dx.doi.org/10.3991/ijoe.v9iS4.2664 \\ Youxin Luo, Qiyuan Liu, Xiaoyi Che , Zheming He \\ Hunan University of Arts and Science, Changde, P.R.China
}

\begin{abstract}
The forward displacement of the generalized parallel mechanism can be converted to nonlinear equations for solving, but it is very difficult to find all solutions because of the strong coupling of the nonlinear equations. Universal grey mathematics was applied to the mechanism synthesis, and based on interval arithmetic toolbox INTLAB the universal grey computing toolbox UG_V1 was compiled. Combining universal grey iterative method with hyperchaotic sequences and taking hyper-chaotic sequences generated by hyper-chaotic neural network system as the initial values of universal grey Newton iterative method, all solutions were found and the calculation steps were given. Example of the generalized parallel mechanism 4SPS-2CCS shows the method is correct and effective. The universal grey method based on hyper-chaos can run within the real range, and it provides a new approach for solving forward displacement in the generalized parallel mechanism and other strongly nonlinear equations.
\end{abstract}

Index Terms-Generalized Stewart parallel mechanism, universal grey interval Newton iterative method, hyperchaotic system, quaternion, nonlinear equations.

\section{INTRODUCTION}

A general 6-6 type parallel mechanism is Stewart mechanism whose upper and lower platforms are flat arbitrary hexagons connected with 6 sliding pairs with spherical pairs at both ends. The position solutions are ultimately boiled down to solving a set of nonlinear equations. That solving these equations is extremely difficult to become another difficult problem of mechanism after completing the displacement analysis in the space $6 \mathrm{R}$ series mechanical arm[1]. If this problem would be broken, the design theory the Stewart platform parallel machine should get a significant development so as to accelerate its application in the mechanical industry, and the solution method should be extended to wider fields to promote the development of a large number of disciplines. The breakthrough of this problem will become an important milestone in solving algebraic equations, the robot operation and the design on machine tool [2]. The basic structure of the traditional Stewart parallel mechanism can be changed by using the hinge distribution and the choice of drive location, to produce a number of variants, but generally speaking, the form of parallel mechanism is relatively simple. X.S. Gao[3] proposed the method constructing new parallel mechanisms by points, lines and surfaces according to angle and distance constraints. New parallel mechanism is named as generalized Stewart parallel mechanism, referred to as generalized parallel mechanism. The calculation shows there are 3850 kinds of mechanisms including the classic Stewart parallel mechanisms in generalized parallel mechanisms which are constructed by new method where there is a mechanism with CCS kinematic chains[4]. The more the CCS kinematic chains in parallel mechanism are, the more complex the forward displacement analysis is [4,5].

The core of the forward displacement is to establish and solve a set of nonlinear constraint equations. The methods solving nonlinear equations in mechanism generally have numerical method and symbolic one. In numerical iteration method or constraint optimization method, if the initial value selected is improper, the result is not easy to convergence more difficult and all solutions are more difficult to get. This problem has not been fully resolved until now, so it has been the research focus of many scholars. Newton iterative method as the traditional numerical iterative method has second-order convergence and high performance, but this method is extremely sensitive to initial value. Sometimes this phenomenon is considered to be the arithmetic singularity or inevitable singularity, in fact, the reason of numerical instability is that NR method is a nonlinear discrete dynamical system where the chaos and fractal phenomena will be generated in the sensitive area. Chaos is called as a phenomenon seemingly irregular and similar to a random in a deterministic system. The most essential characteristics of chaotic behavior is extreme sensitivity to initial conditions of the nonlinear system. Lyapunov exponent is one of effective methods depicting the chaos specific property of nonlinear system. If a system has two or more positive Lyapunov exponents, the system is hyper-chaotic. The more the number of the positive Lyapunov exponents, the higher the degree of instability in the system. It is important theoretical and practical significance that the chaotic and hyper-chaotic systems are used to calculate kinematics. Newton iteration method, the improved Newton iterative method and mathematical programming method based on chaotic sequence in the complex plane mechanism, parallel mechanism and generalized parallel mechanism were researched, and the impact of chaotic sequence characteristics on the solution speed and all solutions were studied [6-9]. The research on the relation between the solving model and the solving speed provides a new approach for the mechanism integration. The interval Newton iteration method based on chaotic sequence was researched [10]. 
With the development of the grey theory, the mathematical foundation of grey system has developed into a new mathematical research areas. Q.Y. Wang etc. creatively put forward the concept of gray set and then standardized the rules of math in interval grey number. But many algebraic properties can not be carried out in the computation of the grey number like the interval mathematics, thus the development is limited. To this end, Q.Y. Wang proposed the concept of universal grey set and laid a basis of universal grey algebra and universal grey mathematics [11]. The universal grey method in the mechanism integration was researched [12].

Universal grey mathematics $[11,12]$ was applied to the mechanism synthesis, and based on interval arithmetic toolbox INTLAB the universal grey computing toolbox UG_V1 was compiled. Combining universal grey iterative method with hyper-chaotic sequences and taking hyperchaotic sequences generated by hyper-chaotic neural network system as the initial values of universal grey Newton iterative method, all solutions were found and the calculation steps were given. Example of the generalized parallel mechanism 4SPS-2CCS shows the method is correct and effective. The universal grey method based on hyper-chaos can run within the real range, and it provides a new approach for solving forward displacement in the generalized parallel mechanism and other strongly nonlinear equations.

\section{GREY MATHEMATICS BASIS}

\section{A. Four Basic operational Rules of the Universal Grey Number}

The concept of Grey number and are seen in references $[11,12]$. Four basic operational rules of the universal grey number are as follows[11,12].

$$
\begin{gathered}
g_{1}+g_{2}=\left(x_{1}+x_{2},\left[\frac{x_{1} \underline{\mu}_{1}+x_{2} \underline{\mu_{2}}}{x_{1}+x_{2}}, \frac{x_{1} \bar{\mu}_{1}+x_{2} \bar{\mu}_{2}}{x_{1}+x_{2}}\right]\right) \\
g_{1}-g_{2}=\left(x_{1}-x_{2},\left[\frac{x_{1} \underline{\mu}_{1}-x_{2} \underline{\mu_{2}}}{x_{1}-x_{2}}, \frac{x_{1} \bar{\mu}_{1}-x_{2} \bar{\mu}_{2}}{x_{1}-x_{2}}\right]\right) \\
g_{1} \times g_{2}=\left(x_{1} x_{2},\left[\underline{\mu}_{1} \underline{\mu_{2}}, \bar{\mu}_{1} \bar{\mu}_{2}\right]\right) \\
g_{1} / g_{2}=\left(x_{1} / x_{2},\left[\underline{\mu}_{1} / \underline{\mu}_{2}, \bar{\mu}_{1} / \bar{\mu}_{2}\right]\right), \text { if } g_{2} \neq g_{(0)}^{\prime}
\end{gathered}
$$

\section{B. Interval Analysis of Universal Grey Mathematics}

The concept of Grey number and are seen in References[11,12] In practical applications, the number $\underline{\mu}$ and $\bar{\mu}$ of $g=(x,[\underline{\mu}, \bar{\mu}])$ is interpreted as the lowest and highest trust level to $x$. A universal grey number can be expressed as an interval number, namely, $(x,[\underline{\mu}, \bar{\mu}])=[x \underline{\mu}, x \bar{\mu}]$. Similarly, the real numbers of the closed interval $\forall[a, b] \in I(R))$ can be expressed as a universal grey number $g=(x,[\underline{\mu}(x), \bar{\mu}(x)])$, at this time $\underline{\mu}, \bar{\mu} \in[-1,1]$ are limited. The conversion rules are seen in references [11,12]. The interval grey number is converted into universal grey number, and ug0.m was written in Matlab2011b. As long as the interval grey number is inputted, the corresponding universal grey number can be found. Universal grey number has the capability of interval analysis and certain advantages. It is proved by the following examples.
Example 1: Find the solution range of a rational function as $f\left(x_{1}, x_{2}, x_{3}\right)=x_{1} \frac{x_{2}+x_{3}}{x_{2}-x_{3}}$, where the variables are expressed by interval numbers and universal grey numbers respectively:

$$
x_{1}=[2,3]=(3,[2 / 3,1]), x_{2}=[1,3]=(3,[1 / 3,1]), x_{3}=[6,18]=(18,[1 / 3,1])
$$

For the original function, the interval number gets the result as $F=[-21,-0.8235]$, and the universal grey number as $F=(-21 / 5,[2 / 3,1])=[-4.2,-2.8]$.

After the original function is deformed as $f\left(x_{1}, x_{2}, x_{3}\right)=x_{1}\left(1+\frac{2}{x_{2} / x_{3}-1}\right)$, the interval number gets the result as $F=[-9,-2.2353]$, and the universal grey number as $F=(-21 / 5,[2 / 3,1])=[-4.2,-2.8]$. The exact solution range of the original function is $[-9,-2.2353]$.

It can be seen in this example that the solution range of the function using the interval number is related to the expression of the function, and there is the relationship as $f([2,3],[1,3],[6,18]) \supseteq[-9,-2.2353]$.

The solution range using the universal grey number has no relation to the expression, but is less than the exact solution when there is the relationship as $f((3[2 / 3,1], 3[1 / 3,1], 18[1,1 / 3]) \subset[-9,-2.2353]$, that is, the solution range using the universal grey number may less than the actual range. It can be found from the universal grey division operation: the upper and lower bounds of the quotient are calculated by the bounds of the universal grey number involved in the calculation, and there is no relation between the upper and lower bounds to make the solution range of the function reduce. Aimed at the universal grey division operation, the following operational rule of the universal grey number was proposed in this paper: if $g_{1}$ and $g_{2}$ are independent of each other, then

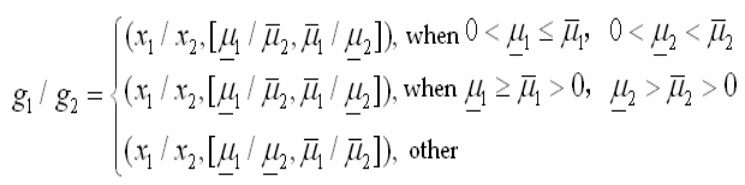

In Example 1, if four basic operational rule of new universal grey number is used, firstly it need to ensure that the numerator and the denominator are independent of each other, so the original function is deformed as $f\left(x_{1}, x_{2}, x_{3}\right)=x_{1}\left(1+\frac{2}{x_{2} / x_{3}-1}\right)$.In the above equation, the interval number was converted to the universal grey number, new operational rule was used to find $F=\left(-\frac{21}{5},\left[\frac{190}{357}, \frac{21}{5}\right]\right)=[-9,-2.2353]$. This result is identical with the exact solution.

Based on the above analysis, the universal grey number not only has the capability of interval analysis, but also can solve this problem not solved in the interval analysis. 
Theorem 1[12] Supposed $F(X)$ is the interval extension function of $f(x)$ in $[a, b]$, if $f(x)=0$ need to has real roots in $[a, b]$, it certainly meets $0 \in F(X)$. If $0 \notin F(X)$, it has no real roots in $[a, b]$.

Because the interval extension is relate to the operation order of the variables, all interval number in the function is converted to the universal grey number, then the above universal grey function is used to calculate.

The real basic elementary functions through the extension of the universal grey is known as the universal grey basic elementary functions that retains the nature of the basic elementary functions, but the interval mathematics does not have these properties. Universal grey numbers has the capability of interval analysis, so it has certain advantages. The universal grey number not only has the capability of interval analysis, but also can solve this problem not solved in the interval analysis. In the mechanism analysis, there are many trigonometric functions. Trigonometric functions in the interval mathematics have no monotonous natural extension while a rational function owns it. So in the mechanism analysis using the interval mathematics or gray number, the domain method or the Taylor series method must be used. The universal grey basic elementary function retains the nature of the basic elementary functions, so it does not have these problems.

\section{Universal Grey Iterative Method}

Based on the universal grey mathematical theory and the above procedures, a universal grey operating toolbox is developed in Matlab environment. The universal grey operating toolbox is based on universal grey class, named as UNIVERSALGREY. INTLAB is the Matlab toolbox for reliable computing and self-validating algorithms. Based on INTLAB v6.0, recompile the uminus, uplus, minus, mtimes, mrdivide and plus functions and so on are developed. The flow chart of the main program is shown in Fig. 1. In this figure, we indicate each Matlab function.

Using a non-interval method it is difficult to find all solutions and often impossible to check whether all solutions have been found. Universal grey interval methods can be used to count the number of roots in a given box and provide tight bounds for each individual root.

Using the mean value theorem we have for any $x^{*}$ that $f\left(x^{*}\right) \in f(\tilde{x})+J(x)\left(x^{*}-\tilde{x}\right)$, where $J(x)$ is the universal grey interval Jacobian matrix with $J_{i j}=\frac{\partial^{2} f}{\partial x_{i} \partial x_{j}}(i, j=1,2, \Lambda, n)$, which can be formed using automatic differentiation, and $\tilde{x} \in X$. If $x^{*}$ is a zero of $\mathrm{f}$ then $f\left(x^{*}\right)=0$ and therefore

$$
f(\tilde{x}) \in J(x)\left(x^{*}-\tilde{x}\right)
$$

The universal grey interval linear system (2) can then be solved for $x^{*}$ to obtain an outer bound on the solution set, say $N(\tilde{x}, x)$. The notation includes both $\tilde{x}$ and $x$ to show the dependence on both terms. This gives $0 \in f(\tilde{x})+J(x)(N(\tilde{x}, x)-\tilde{x})$, which suggests the iteration to solve for $N(\tilde{x}, x)$.

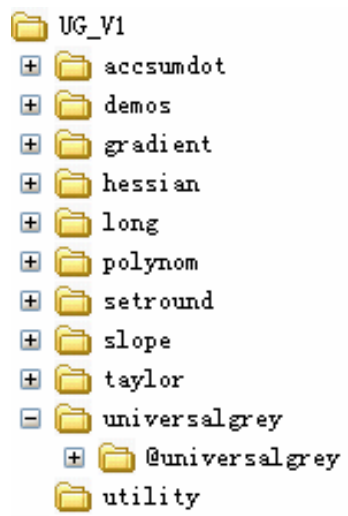

Figure 1. The flow chart of the main program

$$
\begin{array}{r}
f\left(\tilde{x}^{(k)}\right)+J\left(x^{(k)}\right)\left(N\left(\tilde{x}^{(k)}, x^{(k)}\right)-\tilde{x}^{(k)}\right)=0 \\
x^{k+1}=x^{k} \operatorname{I} N\left(\tilde{x}^{(k)}, x^{(k)}\right)
\end{array}
$$

where, $k=0,1, \Lambda$, and $\tilde{x}^{(k)} \in x^{(k)}$. A reasonable choice for $\tilde{X}^{(k)}$ is the centre $\hat{x}^{(k)}$ of $x$. The various universal grey interval Newton methods are determined by how $x^{k+1}=x^{k} \mathrm{I} N\left(\tilde{x}^{(k)}, x^{(k)}\right)$ is defined. The two operators described below are commonly used to find $N(\tilde{x}, x)$. One is Newton operator $N(\tilde{x}, x)=\tilde{x}-J(x)^{-1} f(\tilde{x})$.This operator is not often used since a universal grey interval linear system has to be solved for any iteration. The second is the Krawczyk operator. The advantage of this operator is that no universal grey interval linear equations have to be solved at any stage, thus increasing speed and reliability. The Krawczyk operator is shown as follow.

$$
N(\tilde{x}, x)=\tilde{x}-C f(\tilde{x})-\left(I-C J(x)^{-1}\right)(\tilde{x}-x)
$$

Where $\mathrm{C}$ is the preconditioning matrix, the midpoint inverse of $J(x)$, and $\widetilde{x} \in X$.

Although an inverse is still required, it is the inverse of a real matrix rather than a universal grey interval matrix. The function verifynlss.m is the UG function for finding verified bounds on the roots of nonlinear equations which is based on verifynlss. $m$ in INTLAB interval toolbox. The method is based on the Krawczyk operator with modifications for enclosing the error with respect to an approximate solution. An approximation of the root is provided by the user before a floating point Newton iteration is carried out to give a closer approximation to a root. With the new approximation, an universal grey interval iteration is carried out using a residual version of the Krawczyk operator [10].

\section{UNIVERSAL GREY ITERATIVE METHOD BASED ON HYPER-CHAOTIC SEQUENCES}

The steps of finding the solutions of nonlinear equations based on hyper-chaotic system are as follows: 
(1) According to hyper-chaotic system to establish the hyper-chaotic set $\mathbf{x}(i, j)(i=1,2 ., \Lambda, n, \mathrm{n}$ is variable number, $j=1,2, \Lambda, N, N$ is the length of chaotic set, adjusting length $N$ according to the tried solutions.

(2) If the interval of variable $\mathbf{x}$ is in interval $[a, b]$, transform $\mathbf{X}$ into universal grey interval number, chaotic set $\mathbf{x}(i, j)$ is mapped into the Universal Grey interval to produce the initial value $\mathbf{x}_{0}(i, j)$ of $\mathrm{jth} \mathbf{x}(:, j)$;

(3) Take the initial value of $\mathbf{x}_{0}(i, j)$ as the initial value of universal grey iterative method, call function verifynlss.m for $\mathrm{N}$ times to get all solutions $\mathbf{x}^{*}$.

\section{NUMERICAL EXAMPLE}

In references $[4,8]$,there are ten equations (1), (4), (8)(10) which are the quaternion expression of forward displacement, i.e. the objective equations. When given the every input of parallel mechanism, $g_{0}, g_{1}, g_{2}, g_{3}$,

$q_{0}, q_{1}, q_{2}, q_{3}, x_{1}, x_{2}$ in the objective equations are unknown and other parameters are known. Shown as in the Fig.2, given A1 $(0,0,0)$, A2(-1.8301, 3.1699,-0.5), A3(3.1699, 11.8301, 0.5), A4(6.8301, 11.8301, -1), A5(11.8301, 3.1699,0), A6(10,0,-1), B1 (0, 0, 0), B2(2,3.6049, 0.0693), B3 (-0.8349, 5.4247, -0.9359), B4(3.1651, 5.6835, 0.03), B5(4.3302, 3.3462, -0.8966), $\mathrm{B} 6(2.3302,0.2589,0.9659), \mathrm{V} 1=(0.965923,-0.25883,0)$, V2 $=(0.6,0.8,0), \mathrm{L} 1=9.8080, \mathrm{~L} 2=10.8465, \mathrm{~L} 3=11.0578$, L4 $=12.8826$, L5 =9.6551,L6 =12.3934.

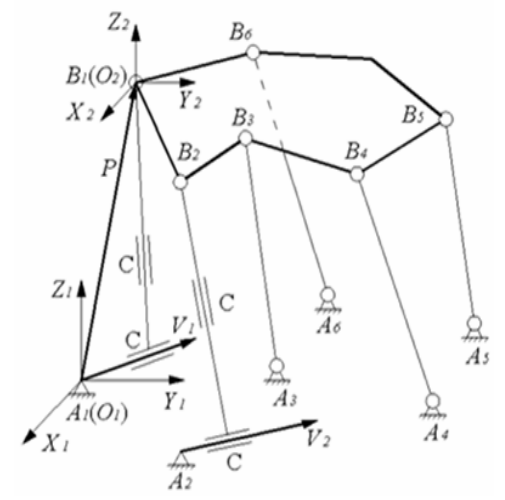

Figure 2. Geometry model of generalized 4SPS-2CCS parallel mechanism

Based on the proposed method, taking the random numbers as the initial points in quaternary hyper-chaotic neural network set[6], the hyper-chaotic system generates the positive Lyapunov exponents hyper-chaotic sequences with variable number $n=10$ (Given hyper-chaotic sequences length $\mathrm{N}=600$ from 2600 group hyper-chaotic sequences . if $\mathrm{N}$ is too large, the calculation time is too long and if $\mathrm{N}$ is too small, all real solutions can not be found, so in this kind of mechanism synthesis, we firstly use less value in the trying process and then use larger value, usually adopting (40 80) $\times$ variables number), running verifynlss('myfun', $x$ ) function by with 6843.0371 s, we can get all real solutions in formula (1),(4),(8)-(10) of references [4] as Table 1.
TABLE I.

12 GROUPS OF REAL SOLUTIONS IN NUMERICAL SAMPLE

\begin{tabular}{lrrccr}
\hline No & \multicolumn{1}{c}{$q_{0}$} & \multicolumn{1}{c}{$q_{1}$} & $q_{2}$ & $q_{3}$ & $g_{0}$ \\
\hline 1. & 0.9620 & -0.1580 & -0.2192 & 0.0405 & 0.7593 \\
2. & -0.9094 & 0.0558 & -0.2876 & 0.2954 & -3.3531 \\
3. & 0.4871 & 0.2008 & 0.2909 & -0.7986 & 4.9242 \\
4. & 0.6548 & 0.5709 & -0.0591 & -0.4917 & 3.4063 \\
5. & -0.9620 & 0.1580 & 0.2192 & -0.0405 & -0.7593 \\
6. & -0.6548 & -0.5709 & 0.0591 & 0.4917 & -3.4063 \\
7. & 0.9094 & -0.0558 & 0.2876 & -0.2954 & 3.3531 \\
8. & 1.0000 & 0.0000 & 0.0000 & -0.0000 & 0.0002 \\
9. & -0.9772 & 0.0900 & 0.1699 & -0.0906 & 0.4266 \\
10. & 0.9772 & -0.0900 & -0.1699 & 0.0906 & -0.4266 \\
11. & -0.4871 & -0.2008 & -0.2909 & 0.7986 & -4.9242 \\
12. & -1.0000 & -0.0000 & -0.0000 & 0.0000 & -0.0002 \\
\hline No & $g_{1}$ & $g_{2}$ & $g_{3}$ & $x_{1}$ & $x_{2}$ \\
\hline 1. & 10.0329 & -2.5305 & 7.4116 & 8.1471 & 4.3524 \\
2. & 1.0768 & 1.3361 & -9.2263 & 1.7651 & 1.9583 \\
3. & -5.0059 & 5.7593 & 3.8427 & 1.0225 & 6.0774 \\
4. & -3.1074 & 8.8049 & -0.1300 & -1.6138 & 6.1506 \\
5. & - & & & & \\
6. & 10.0329 & 2.5305 & -7.4116 & 8.1471 & 4.3524 \\
7. & -1.0768 & -1.3361 & 9.2263 & 1.7651 & 1.9583 \\
8. & 3.8341 & -0.8184 & 9.8061 & 3.9156 & 1.8919 \\
9. & -8.6647 & 2.6658 & -8.2148 & 7.3248 & 3.0279 \\
10. & 8.6647 & -2.6658 & 8.2148 & 7.3248 & 3.0279 \\
11. & 5.0059 & -5.7593 & -3.8427 & 1.0225 & 6.0774 \\
12. & -3.8341 & 0.8184 & -9.8061 & 3.9156 & 1.8919 \\
\hline & & & & &
\end{tabular}

\section{CONCLUSIONS}

Using quaternary hyper-chaotic neural network to generate initial points of universal grey interval Newton iterative methods, we can find all the real solutions of nonlinear equations for 4SPS-2CCS parallel mechanism in real number range. As the solution is in the real number range, the calculation efficiency is high. The numerical example of forward displacement for generalized 4SPS2CCS parallel mechanism denotes the method is correct and effective. This method is simple and can be widely used in other forward displacement for parallel mechanism. It provides a theoretic foundation for the dimensional design and path programming for this kind mechanism.

\section{ACKNOWLEDGMENT}

This research is supported by the National Natural Science Foundation of P.R. China (No:51075144) and the grant of the 12th Five-Year Plan for the construct program of the key discipline (Mechanical Design and Theory) in Hunan province(XJF2011[76]) and Hunan Provincial Natural Science Foundation of China(No:13JJ8023).

\section{REFERENCES}

[1] F. A. Wen, C. G. Liang, Q.Z. Liao, "The forward displacement analysis of parallel robotic mechanisms", China Mechanical Engineering, Vol.10, Issue 9,1999, pp.1011-1013.

[2] X.X. Li, 100 Interdisciplinary science puzzles of the 21st century, China Science Press, Beijing, 2005.

[3] X. S. Gao, D.L. Lei,Q. Z. Liao, G.F. Zhang, "Generalized StewartGough platforms and Their Direct Kinematics", IEEE Transactions on Robotics, Vol.21, Issue 2, 2005 ,pp.141-151.

[4] X.G. Huang, Q.Z. Liao, S.M. Wei, et a1., "Forward Displacement Analysis of the 4SPS-2CCS Generalized Stewart Platform", Journal of Beijing University of Posts and Telecommunications, Vol.30, Issue 3, 2007, pp.5-18,31.

[5] X.G. Huang, Q.Z. Liao, D.L. Li, S.M. Wei, "Forward Displacement Analysis of Generalized 5SPS-1CCS Parallel Robot 
Mechanism Based on Quaternion”, Journal of Mechanical Engineering, Vol.43, Issue 5, 2007, pp.8-13. http://dx.doi.org/ 10.3901/JME.2007.05.008

[6] Y.X. Luo, Q.Y. Liu, X.Y. Che, B. Zeng, "Forward displacement analysis of the 6-SPS Stewart mechanism based on quaternion and hyper-chaotic damp least square method", Advanced Materials Research, Vol.230-232, 2011,pp. 759-763. http://dx.doi.org/ 10.4028/www.scientific.net/AMR.230-232.759

[7] Y.X. Luo, X.Y. Che, B. Zeng, "Displacement analysis of the 3SPS-3CCS mechanism based on hyper-chaotic Newton-downhill method, Key Engineering Materials, Vol. 467-469, 2011,pp. 401406. http://dx.doi.org/10.4028/www.scientific.net/KEM.467469.401

[8] Y.X. Luo, X.Y. Che, B. Zeng, "Displacement analysis of the 4SPS-2CCS mechanism based on hyper-chaotic Newton-downhill method", Advanced Materials Research, Vol. 230-232, 2011,pp. 733-737. http://dx.doi.org/10.4028/www.scientific.net/AMR.230$\underline{232.733}$

[9] Y.X. Luo, X.Y. Che, B.Zeng, "Forward Displacement Analysis of the 2CCC-4SPS Generalized Stewart Parallel Robot Mechanism Based on Chaotic Anti-Control Damping Least Squares Method", Advanced Science Letters, Vol.19, Issue.8, 2013,pp. 2387-2390. http://dx.doi.org/10.1166/asl.2013.4914

[10] Y.X. Luo, X.Y. Che, C.Wang, "Displacement Analysis of the 3SPS-3CCS Mechanism Hyper-Chaotic-Based Interval Iteration Method", Advanced Science Letters, Vol.19, Issue 8, 2013,pp. 2456-2459. http://dx.doi.org/10.1166/asl.2013.4913
[11] Q.Y.Wang, "Foundations of Universal Grey Algebra”, Journal of Huazhong University of Science and Technology, Vol.20, Issue 4, 1992 , pp.151-156.

[12] Y.X.Luo, Z.M. He, H.X. Guo, L.T.Zhuang, "Application of Universal Grey Number to Synthesis of Plane Rigid Guidance", Journal of Mechanical Transmission, Vol.26, Issue.2, 2002, pp.73-75

\section{AUTHORS}

Youxin LUO is with College of Mechanical Engineering, Hunan University of Arts and Science, Changde, P.R. China (E_mail:LLYX123@126.com).

Qiyuan Liu is with College of Mechanical Engineering, Hunan University of Arts and Science, Changde, P.R. China.

XiaoYi CHE is with College of Mechanical Engineering, Hunan University of Arts and Science, Changde, P.R. China.

Zheming He is with College of Mechanical Engineering, Hunan University of Arts and Science, Changde, P.R. China.

This article is an extended and modified version of a paper presented at the International Conference on Mechanical Engineering, Automation and Material Science (MEAMS2012), held 22-23 December 2012, Wuhan, China. Received 06 January 2013. Published as resubmitted by the authors 01 May 2013. 